\title{
The corticomotor projection to liminally-contractable forearm muscles in chronic spinal cord injury: a transcranial magnetic stimulation study
}

\author{
M Cortes ${ }^{1,2,3}$, GW Thickbroom ${ }^{1,4}$, J Elder ${ }^{5}$, A Rykman ${ }^{1}$, J Valls-Sole ${ }^{3}$, A Pascual-Leone ${ }^{6,7}$ and DJ Edwards R $^{1,2,6}$
}

Study design: A cross-sectional study in chronic spinal cord injury with cervical lesions (cSCl).

Objective: To determine the corticomotor projection and motor cortex organization of paralyzed forearm muscles that presented only liminal voluntary activation.

Setting: Burke Medical Research Institute, White Plains, NY, USA.

Methods: We identified ten people with chronic SCI who had a wrist flexor or extensor muscle with a motor power (MP) of 1 over 5. We recorded motor evoked potentials (MEPs) to transcranial magnetic stimulation (TMS) over the primary motor cortex of the hemisphere contralateral to the target muscle. We measured resting motor threshold (RMT), corticomotor latency (LTY), MEP amplitude (AMP) and performed cortical motor mapping to determine the optimal site (OPT) and map area (AREA). Results were compared with the data from 18 controls.

Results: A MEP in the target muscle was observed for all CSCI cases. LTY was normal, while corticomotor excitability (as determined by RMT and AMP) was reduced in about half of the group. The OPT site of the motor maps was within control range for all cSCl cases, while AREA was reduced in three cases.

Conclusions: Corticomotor conduction and cortical topography were appreciably normal despite only liminal activation of the target muscle with voluntary effort. Muscles with these characteristics may benefit from a targeted rehabilitation program even in the chronic phase after $\mathrm{SCl}$.

Spinal Cord (2017) 55, 362-366; doi:10.1038/sc.2016.161; published online 20 December 2016

\section{INTRODUCTION}

A cervical spinal cord injury ( $\mathrm{CSCI}$ ) can result in paralysis or severe motor deficits in the upper and lower extremities that can have a devastating impact on independence and quality of life. ${ }^{1}$ In the upper extremities, recovery of function in the wrist flexors and extensors is important as it can lead to improvements in arm and hand function. ${ }^{2,3}$

The American Spinal Injury Association (ASIA) Impairment Scale (AIS) is widely used to classify impairment (motor and sensory) after spinal cord injury. For the assessment of upper limb motor power, the AIS scale uses the Medical Research Council (MRC) scale for motor power (MP) and pools this for 5 functionally relevant movements (including wrist extension). The MRC scale ranges from 0 to 5 , with a MP of 1 defined as a trace of muscle contraction or fasciculation, but no overt movement about the joint.

We recently described a cSCI case study in which corticospinal conduction to the wrist flexors and extensors was remarkably preserved, even though wrist MP was only 1 over $5 .{ }^{4}$ Using transcranial magnetic stimulation (TMS), we reported motor evoked potentials (MEPs) of normal amplitude (AMP) and latency for these wrist muscles. This observation suggested that supraspinal motorrelated networks could be implicated in functional weakness after cSCI. ${ }^{5,6}$ However, the changes that might occur in these networks in response to cSCI are not well understood.

It is known that spinal cord injury can result in cortical atrophy ${ }^{7-9}$ and there is evidence for cortical reorganisation from functional magnetic resonance imaging ( $\mathrm{fMRI}$ ), positron emission tomography (PET) and electroencephalographic (EEG) studies. ${ }^{10,11}$ TMS can be used to map the corticomotor projection from primary motor cortex (M1) to a target muscle ${ }^{12}$ and provides another opportunity to investigate cortical reorganisation. While TMS mapping has been used to identify reorganisation of muscles innervated above the level of the lesion, ${ }^{10,13}$ there have been no such studies of severely impaired muscles at the level of the lesion.

In the present study, we have measured corticomotor conduction and performed TMS mapping in a group of 10 people with cSCI who presented with muscle strength in a forearm muscle of 1 over 5 . Our aims were to determine how corticomotor conduction was affected in

${ }^{1}$ Non-invasive Brain Stimulation and Human Motor Control Laboratory, Burke Medical Research Institute, White Plains, NY, USA; ${ }^{2}$ Department of Neurology, Cornell University, New York, NY, USA; ${ }^{3}$ EMG and Motor Control Unit, Neurology Department, Hospital Clinic, Universitat de Barcelona, Barcelona, Spain; ${ }^{4}$ Department of Rehabilitation Medicine, Cornell University, New York, NY, USA; ${ }^{5}$ Department of Epidemiology, Cornell University, New York, NY, USA; ${ }^{6}$ Berenson-Allen Center for Noninvasive Brain Stimulation, Division of Cognitive Neurology, Beth Israel Deaconess Medical Center, Harvard Medical School, Boston, MA, USA and ${ }^{7}$ Institut Universitari de Neurorehabilitacio Guttmann, Department of Neurology, Universitat Autonoma, Barcelona, Spain

Correspondence: Dr M Cortes, Non-invasive Brain Stimulation and Human Motor Control Burke Medical Research Institute, 785 Mamaroneck Avenue, White Plains 10605 , NY, USA.

E-mail: mac2083@med.cornell.edu

Received 29 March 2016; revised 22 July 2016; accepted 26 September 2016; published online 20 December 2016 
Table $1 \mathrm{SCl}$ participant details

\begin{tabular}{|c|c|c|c|c|c|c|c|c|}
\hline No. & Gender & Age (years) & Level & AIS & TSI (years) & Muscle & Side & $M P$ \\
\hline 1 & $\mathrm{~F}$ & 29 & $\mathrm{C} 4$ & $B$ & 2.3 & FCR & $\mathrm{L}$ & 1 \\
\hline 2 & M & 31 & C5 & $B$ & 7.5 & FCR & $\mathrm{L}$ & 1 \\
\hline 3 & M & 44 & $\mathrm{C} 4$ & C & 1.8 & ECR & $\mathrm{R}$ & 1 \\
\hline 4 & $\mathrm{~F}$ & 55 & $\mathrm{C} 5$ & $B$ & 2.1 & FCR & $\mathrm{R}$ & 1 \\
\hline 5 & M & 54 & C6 & $A$ & 2.2 & FCR & $\mathrm{R}$ & 1 \\
\hline 6 & M & 70 & C4 & C & 3 & ECR & $\mathrm{R}$ & 1 \\
\hline 7 & $\mathrm{~F}$ & 24 & $\mathrm{C} 4$ & B & 5.8 & ECR & $\mathrm{L}$ & 1 \\
\hline 8 & $M$ & 17 & C4 & B & 4 & ECR & $\mathrm{R}$ & 1 \\
\hline 9 & $M$ & 50 & C5 & $A$ & 29 & FCR & $L$ & 1 \\
\hline 10 & $M$ & 50 & C4 & $C$ & 3 & ECR & $L$ & 1 \\
\hline
\end{tabular}

Abbreviations: AIS, Asia impairment scale; ECR, extensor carpi radialis; F, female; FCR, flexor carpi radialis; M, male; MP, motor power; TSI, time since injury.

Age (years); TSI (years).

people with a MP of $1 / 5$, and to determine whether the cortical maps were normal or showed signs of reorganisation.

\section{METHODS}

\section{Participants}

Ten people with cSCI volunteered to participate in the study (Table 1). All met the following inclusion criteria: age 18-70 years, chronic injury ( $>1$ year after injury), cervical injury level, traumatic lesion, motor complete or incomplete, MP of wrist extensor or flexor muscles $1 / 5$, no evidence of trauma-related brain injury, no contraindications for TMS, no history of seizure and medically stable. A second investigator independently confirmed the MP score. All cSCI participants were right-handed prior to injury and remained so thereafter. None of the participants had a history of neurological or psychiatric disorders. Eighteen right-handed healthy participants (24-51 years of age, 8 male) without a history of neurological or psychiatric illness were recruited as a control group. The Institutional Review Board of the Burke Rehabilitation Hospital approved the experimental protocol, and all subjects gave written informed consent prior the experiment.

\section{Motor power}

The MP of the Extensor Carpi Radialis (ECR) and Flexor Carpi Radialis (FCR) muscles on the left and right sides was scored independently by two neurologists. The muscle identified by both scorers as having a MP of 1 was the target muscle for the remainder of the study.

\section{EMG Recording}

Bipolar surface electromyography (EMG) electrodes $(1 \mathrm{~cm}$ diameter, $2 \mathrm{~cm}$ inter-pole distance, $\times 1000$ gain, band-pass filter $20-400 \mathrm{~Hz}$ ) were placed over the belly of the target muscle. EMG activity was recorded by Biometrics electromyography (Biometrics Ltd, UK), and signals were fed into CED 1401 using Spike 2.6 for further off-line analysis. During the experiments, EMG activity was continuously monitored with visual feedback to ensure complete muscle relaxation.

An estimate of maximum voluntary contraction (MVC) in the cSCI group was obtained by recording short periods of EMG, while the participant attempted a maximum muscle contraction. Three $0.5 \mathrm{~s}$ epochs of EMG were acquired, and the average root-mean-square EMG calculated. Three $0.5 \mathrm{~s}$ epochs of resting EMG were also acquired as an estimate of signal noise.

TMS measurements were taken for the ECR and FCR muscles on the left and right sides in the 18 controls.

\section{Transcranial magnetic stimulation (TMS)}

SCI participants were seated in their own wheelchairs and wore a snugly-fitting pre-marked cap with sites marked in spacings of $1 \mathrm{~cm}$ in latitude and $2 \mathrm{~cm}$ in longitude in relation to the vertex and inter-aural line. ${ }^{12,14} \mathrm{~A}$ figure-of-eight coil (DB-80, Tonika Elektronik A/S, DK-3520, Denmark) was connected to a MagPro magnetic stimulator (MagVenture, Farum, Denmark). The coil was manually positioned on the scalp over the expected location of the contralateral primary motor cortex (M1), with the handle pointing backwards at an angle of 45 degrees to the midline. ${ }^{15}$ The optimal site was established by a search pattern at suprathreshold intensity to determine the location yielding the largest MEPs. Resting motor threshold (RMT) was measured at this site and was defined as the minimum intensity ( $2 \%$ steps in stimulator output) required to elicit at least $3 \mathrm{MEPs}$ with a peak-peak AMP $>50 \mu \mathrm{V}$ in 5 consecutive trials. ${ }^{16,17}$ For the remainder of the experiment, TMS intensity was set to $1.2 \times$ RMT. Twenty MEPs were recorded at the optimal site at this intensity. MEP peak-peak AMP and MEP onset latency (LTY) were determined from these data by manual cursoring and averaged for each muscle.

\section{Motor cortex mapping}

Five stimuli were delivered at a frequency of $0.2 \mathrm{~Hz}$ at each stimulation site starting at the optimal site and then successively stimulating adjacent sites until no MEP was found ( $<50 \mu \mathrm{V}$ AMP). To maintain consistency in coil orientation as it was repositioned over the scalp for mapping, the coil was held in the parasagittal plane. Maps were generated by fitting a continuous function to the mean peak-to-peak MEP AMP at each scalp site (Wilson 1993). The latitude (LAT) and longitude (LONG) at which the map had its maximum AMP was determined. LAT was expressed as the distance in centimeters from the vertex, and LONG the distance in centimeters anterior (positive) or posterior (negative) to the inter-aural line. To avoid biasing map area (AREA) by small MEPs on the periphery, AREA was calculated from the region of the map with an AMP $>1 / 8$ of maximum.

\section{Statistical analysis}

A Wilcoxon rank-sum was used to test for an effect of SIDE (left, right) and MUSCLE (ECR, FCR). A two-way ANOVA was used to assess for an interaction between SIDE and MUSCLE.

From the control data, 95\% confidence intervals were determined for each parameter (RMT, AMP, LTY, LAT, LONG, AREA). The lower limit (LL) was 2 s.d. below the mean and the upper limit (UL) was 2 s.d. above the mean. The AMP data was skewed and log transforms were used to establish LL and UL. The SCI data for each participant was compared with control ranges.

\section{RESULTS}

SCI subject's characteristics are given in Table 1 . The mean age was $42.4 \pm 16.5$ years (s.d.; range $17-70$ years of age; $7 \mathrm{M}, 3 \mathrm{~F}$ ). The mean period following injury was $6.1 \pm 8.2$ years (range 1.8-29 years). Eight of the SCI group had incomplete lesions and two had complete lesions. Two subjects were classified as motor-sensory complete (AIS A), five as motor complete but sensory incomplete (AIS B), and three as motor and sensory incomplete (one as AIS C). All participants had severe upper and lower limb impairment. Across the SCI group, the target muscles that had a MP of 1 were evenly split between left and right sides (five cases each) and ECR and FCR (also five cases for each).

The mean age of the control group was $36.1 \pm 6.8$ (s.d., range 24-51 years). A Wilcoxon rank-sum test revealed no effect of SIDE (for all parameters) but an effect of MUSCLE for most parameters $(P<0.05)$. As a result, the data was pooled for the left and right sides and control ranges (LL, UL) established separately for the ECR and FCR (see Table 2).

For the SCI group, an analysis using two-way Anova revealed there were no effects for SIDE, MUSCLE or SIDE x MUSCLE. Correspondingly, the SCI data from either side (left or right) was compared with the control ranges for ECR and FCR separately.

\section{Corticomotor excitability and conduction}

A MEP was observed for all cases in the SCI group. The LTY was within control range for all but one of the SCI cases. For all five SCI cases, in whom the ECR was studied, RMT and AMP were outside 
the control ranges and there was a significant difference between this SCI subgroup and controls ( $t$-test, $P<0.001$ and $P=0.003$, respectively). For the five cases in whom the FCR was studied, RMT and AMP were within the control ranges for all but one case and there was a significant difference between this subgroup and controls for RMT $(P<0.02)$. There was no significant difference between ECR or FCR for RMT or for AMP $(P=0.7$ and $P=0.4$, respectively). The RMS ranged from 0.008 to $0.017 \mathrm{mV}$ for ECR and 0.007 to $0.015 \mathrm{mV}$ for FCR (mean for combined muscles $0.011 \pm 0.004 \mathrm{mV}$ ). Mean resting RMS was $0.007 \pm 0.002 \mu \mathrm{V}$. There was no correlation between RMS during an attempted MVC and $\operatorname{AMP}(P=0.22)$.

Table 2 The control group data

\begin{tabular}{|c|c|c|c|c|c|c|}
\hline & \multicolumn{3}{|c|}{$E C R$} & \multicolumn{3}{|c|}{ FCR } \\
\hline & Mean & $L L$ & $U L$ & Mean & $L L$ & $U L$ \\
\hline RMT & 32.9 & 23.9 & 42 & 42.4 & 26.3 & 58.4 \\
\hline AMP & 560 & 184 & 1699 & 299 & 112 & 799 \\
\hline LTY & 16.3 & 13.6 & 19.0 & 17.1 & 14.6 & 19.6 \\
\hline LAT & 5.4 & 3.2 & 7.6 & 5.7 & 2.6 & 8.7 \\
\hline LONG & -0.7 & -5.2 & 3.7 & -0.5 & -4.7 & 3.7 \\
\hline AREA & 47.0 & 19.9 & 74.0 & 47.0 & 28.4 & 65.5 \\
\hline
\end{tabular}

Abbreviations: AMP, amplitude; AREA, map area; ECR, extensor carpi radialis; FCR, flexor carp radialis; LAT, latitude; LL, lower limit; LONG, longitude; LTY, corticomotor latency; RMT, resting motor threshold; UL, upper limit.

Neurophysiological outcomes mean values are stated as well as the lower and upper limit, expressed as 2 s.d. of the mean ( 2 s.d.), for the forearm muscles (ECR and FCR).

\section{Corticomotor mapping}

The TMS maps could be obtained for all SCI cases. Figure 1 gives MEP waveforms and maps for a representative case from the SCI group and the control group. Table 2 gives the control ranges for each parameter and Table 3 provides the results for each case in the SCI group.

The LAT and LONG were within the control range for all SCI cases (Figure 2). The AREA was reduced in the FCR for three cases (two marginally).

Table 3 The SCl individual data

\begin{tabular}{lcccccccc}
\hline No. & Muscle & RMT & AMP & LTY & RMS & LAT & LONG & AREA \\
\hline 1 & FCR & 40 & 183 & 15.9 & 0.007 & 3.7 & -1.3 & $27.4^{\mathrm{b}}$ \\
2 & FCR & 58 & 127 & 17.3 & NA & 3.6 & 0.7 & $20.8^{\mathrm{b}}$ \\
3 & ECR & $44^{\mathrm{a}}$ & $159^{\mathrm{b}}$ & $19.9 \mathrm{a}$ & 0.008 & 4.9 & -3.1 & 31.4 \\
4 & FCR & 38 & 279 & 15.8 & 0.015 & 5.4 & -1.7 & 33.9 \\
5 & FCR & 55 & 121 & 16.9 & 0.008 & 3.5 & 1.7 & $27.6^{\mathrm{b}}$ \\
6 & ECR & $44^{\mathrm{a}}$ & $161^{\mathrm{b}}$ & 15.6 & 0.014 & 3.7 & 0.7 & 34.3 \\
7 & ECR & $45^{\mathrm{a}}$ & $145^{\mathrm{b}}$ & 16.6 & 0.006 & 6.7 & -2.5 & 50.4 \\
8 & ECR & $56^{\mathrm{a}}$ & $134^{\mathrm{b}}$ & 18.5 & 0.008 & 4.0 & -2.6 & 35.9 \\
9 & FCR & $62^{\mathrm{a}}$ & 189 & 17.7 & NA & 6.2 & -1.0 & 35.5 \\
10 & ECR & $80^{\mathrm{a}}$ & $168^{\mathrm{b}}$ & 14.7 & 0.018 & 3.9 & 0.6 & 33.7 \\
\hline
\end{tabular}

Abbreviations: AMP, amplitude; AREA, map area; ECR, extensor carpi radialis; FCR, flexor carpi radialis; LAT, latitude; LONG, longitude; LTY, corticomotor latency; NA, not available; RMS, root mean square; RMT, resting motor threshold.

AMP $(\mu \mathrm{V})$; AREA $\left(\mathrm{cm}^{2}\right)$; LAT (in $\mathrm{cm}$ ); LONG (in $\mathrm{cm}$ ); LTY (ms); RMS (root mean square EMG during MVC); RMT (\% stimulator output); '<' below control LL; '>' above control UL

${ }^{a}$ Above control UL.

${ }^{\mathrm{b}}$ Below control LL.
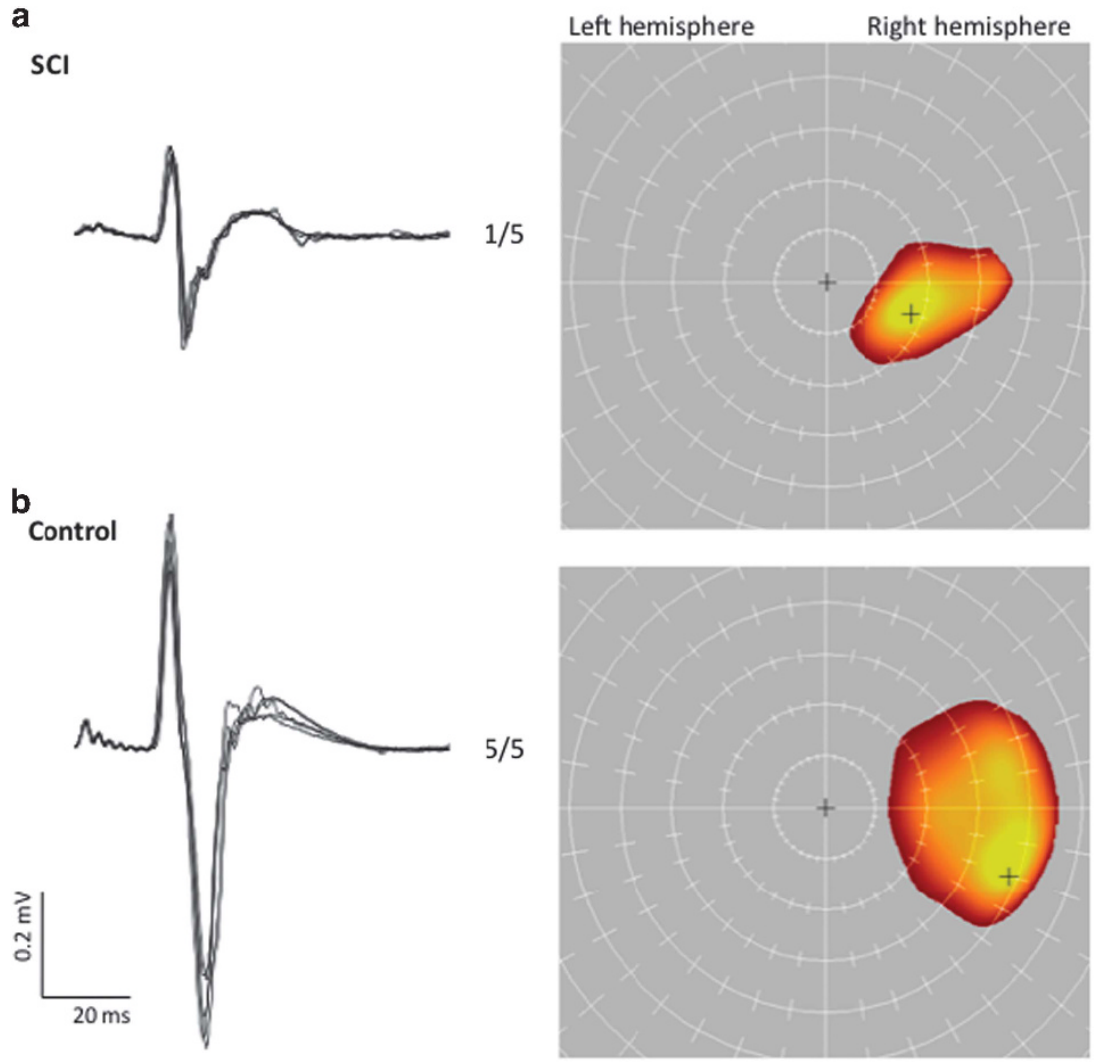

Figure 1 The MEP waveforms and maps for a representative case from the $\mathrm{SCl}$ group and the control group. 

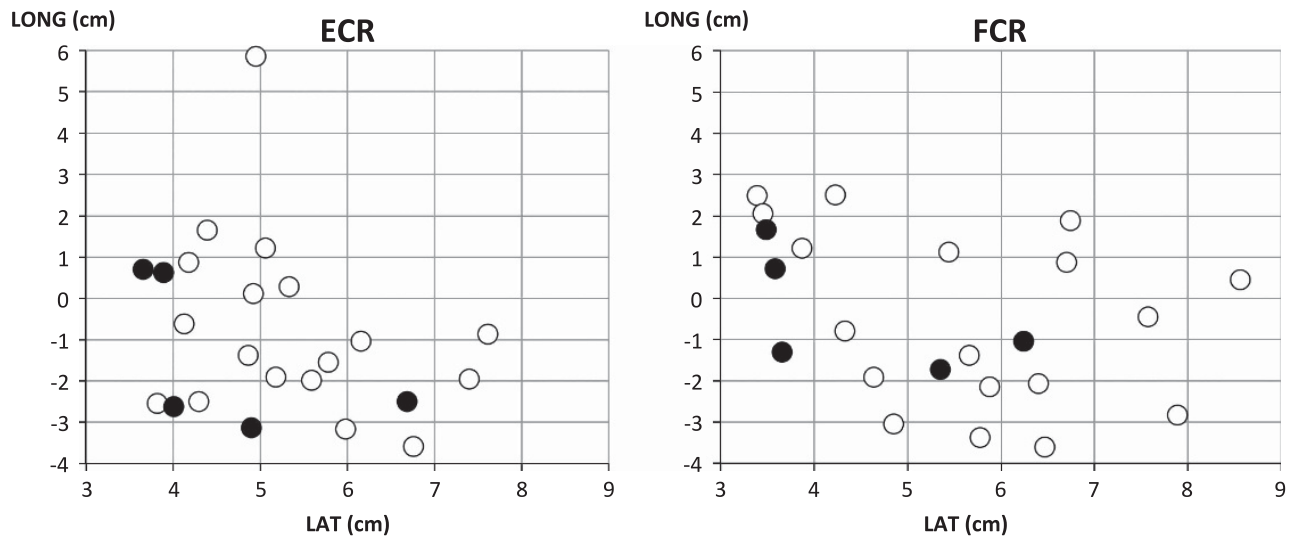

Figure 2 The individualized data showing that the maps characteristics (LAT and LONG) were within control range (open circles) for all SCl cases (filled circles).

\section{DISCUSSION}

It was possible to determine a RMT, record a MEP and measure a motor map for a forearm muscle in all cases from this group of chronic cervical SCI. The unifying feature in the group was the selection of a target muscle with a MP of $1 / 5$. We showed that corticomotor conduction through the level of the spinal injury is remarkably patent and that the topographic origins of this projection in M1 are normal. The results indicate that in the chronic phase after injury, and in muscles with only liminal voluntary activation, there can remain a significant neurophysiological substrate that could be a target for rehabilitation.

We have previously reported that corticomotor conduction can be within control values in a case study of chronic cervical SCI. ${ }^{4,18}$ The present study generalises this finding by showing that it is not rare to find patent corticomotor conduction in muscles with only liminal levels of voluntary activation in the chronic phase after SCI.

Corticomotor conduction time was within control range for all but one SCI case that was marginally delayed. This is in contrast to some previous studies that indicate conduction through the lesion is often delayed. ${ }^{19-21}$ However, spinal cord injury is expected to have diverse effects on corticospinal conduction depending on the nature of the lesion. In our cSCI group, selected to have a degree of commonality (chronic and stable, cervical lesions, forearm wrist extensors/flexors, $M P=1$ ), we find that the fastconducting monosynaptic pathway to the target muscles can be relatively normal.

While RMT was increased and AMP decreased in about half of the cases, in many these data were not greatly outside of control range. Across the group, we mostly observed these abnormalities for the ECR rather than the FCR muscle. In the control group, we found intermuscle differences, with the ECR having a lower RMT and higher AMP than the FCR. This difference was not seen in the SCI group, presumably because RMT was increased and AMP reduced mostly just for the ECR muscle. The reason for this is not certain; however, it could be related to differences in the corticospinal projection to wrist flexor and extensor muscles. It is known that wrist extension can be severely affected in $\mathrm{SCI}^{22}$ and the wrist extensors are target muscles in the AIS scoring system. ${ }^{23}$

The retention of corticospinal conduction through the lesion is consistent with anatomical studies that show there are usually spared axons across a lesion. ${ }^{24-26}$ However, less is known about the corticomotor pathways these axons serve. The TMS mapping data suggest that the spared axons that give rise to the MEP originate from the expected forearm representation in M1. It is known that the cerebral cortex is remarkably adaptable, and that cortical and subcortical lesions can lead to functional reorganisation. ${ }^{27-29}$ Previous studies of cortical reorganisation after SCI have mostly employed functional imaging techniques such as functional MRI and PET. ${ }^{11}$ These studies require voluntary activation of a target limb, often targeting muscles proximally to the lesion, which can recruit multiple brain regions and involve polysynaptic pathways not accessible to TMS. The present study was performed in forearm muscles with $\mathrm{MP}=1$ and indicated that the corticospinal neurons giving rise to the MEP in these muscles had a normal cortical topography. We cannot say whether this would be true in higherfunctioning proximal muscles, where reorganisation might take place as a result of use-dependent plasticity, or in muscles of the lower extremity.

Perhaps the most intriguing finding is that even for those muscles with normal corticomotor conduction and cortical topography, the muscles could only be liminally activated under voluntary control, even when participants were asked to do a maximal effort. The underlying cause of this functional paralysis is not certain and may perhaps involve a learned disuse. If so, the present results increase confidence that clinically meaningful improvement in function might be achieved with a suitable rehabilitation program. The objective data of corticomotor conduction, such as provided by TMS, may also increase the likelihood of success, if communicated to participants.

In conclusion, in our cSCI group, targeting a forearm muscle with $\mathrm{MP}=1 / 5$, corticomotor conduction and cortical topography were appreciably normal despite only liminal activation with voluntary effort. This suggests that muscles with these characteristics may benefit from a targeted rehabilitation program even in the chronic phase after SCI. In the absence of TMS, our data suggests that further rehabilitation of muscles with a MP of 1/5 might be indicated. Demonstration of a good cortical-motor connection by TMS may be a fillip to people struggling with recovery of weakly activated muscles after SCI.

\section{CONFLICT OF INTEREST}

The authors declare no conflict of interest.

\section{DATA ARCHIVING}

There were no data to deposit. 
1 Welch RD, Lobley SJ, O'Sullivan SB, Freed MM. Functional independence in quadriplegia: critical levels. Arch Phys Med Rehabil 1986; 67: 235-240.

2 Ditunno JF Jr, Sipski ML, Posuniak EA, Chen YT, Staas WE Jr, Herbison GJ. Wrist extensor recovery in traumatic quadriplegia. Arch Phys Med Rehabil 1987; 68 (5 Pt 1): 287-290.

3 Hanson RW, Franklin MR. Sexual loss in relation to other functional losses for spinal cord injured males. Arch Phys Med Rehabil 1976; 57: 291-293.

4 Edwards DJ, Cortes M, Thickbroom GW, Rykman A, Pascual-Leone A, Volpe BT. Preserved corticospinal conduction without voluntary movement after spinal cord injury. Spinal Cord 2013; 51: 765-767.

5 Curt A, Dietz V. Electrophysiological recordings in patients with spinal cord injury: significance for predicting outcome. Spinal Cord 1999; 37: 157-165.

6 Iseli E, Cavigelli A, Dietz V, Curt A. Prognosis and recovery in ischaemic and traumatic spinal cord injury: clinical and electrophysiological evaluation. J Neurol Neurosurg Psychiatry 1999; 67: 567-571.

7 Jurkiewicz MT, Crawley AP, Verrier MC, Fehlings MG, Mikulis DJ. Somatosensory cortical atrophy after spinal cord injury: a voxel-based morphometry study. Neurology 2006; 66: 762-764.

8 Nie B, Chen K, Zhao S, Liu J, Gu X, Yao Q et al. A rat brain MRI template with digital stereotaxic atlas of fine anatomical delineations in paxinosspace and its automated application in voxel-wise analysis. Hum Brain Mapp 2013; 34: 1306-1318.

9 Ghosh A, Haiss F, Sydekum E, Schneider R, Gullo M, Wyss MT et al. Rewiring of hindlimb corticospinal neurons after spinal cord injury. Nat Neurosci. 2010; 13: 97-104.

10 Lotze M, Laubis-Herrmann U, Topka H. Combination of TMS and fMRI reveals a specific pattern of reorganization in M1 in patients after complete spinal cord injury. Restor Neurol Neurosci 2006; 24: 97-107.

11 Kokotilo KJ, Eng JJ, Curt A. Reorganization and preservation of motor control of the brain in spinal cord injury: a systematic review. J Neurotrauma 2009; 26: 2113-2126.

12 Wilson SA, Thickbroom GW, Mastaglia FL. Transcranial magnetic stimulation mapping of the motor cortex in normal subjects. the representation of two intrinsic hand muscles. J Neurol Sci 1993; 118: 134-144.

13 Freund P, Rothwell J, Craggs M, Thompson AJ, Bestmann S. Corticomotor representation to a human forearm muscle changes following cervical spinal cord injury. Eur $J$ Neurosci 2011; 34: 1839-1846.

14 Thickbroom GW, Byrnes ML, Mastaglia FL. Methodology and application of TMS mapping. Electroencephalogr Clin Neurophysiol Supp/ 1999; 51: 48-54.

15 Brasil-Neto JP, Cohen LG, Panizza M, Nilsson J, Roth BJ, Hallett M. Optimal focal transcranial magnetic activation of the human motor cortex: effects of coil orientation, shape of the induced current pulse, and stimulus intensity. J Clin Neurophysiol 1992; 9: 132-136.
16 Rossi S, Hallett M, Rossini PM, Pascual-Leone ASafety of TMS Consensus Group Safety, ethical considerations, and application guidelines for the use of transcranial magnetic stimulation in clinical practice and research. Clin Neurophysiol 2009; 120: 2008-2039.

17 Rothwell JC, Hallett M, Berardelli A, Eisen A, Rossini P, Paulus W. Magnetic stimulation: motor evoked potentials. The International Federation of Clinical Neurophysiology. Electroencephalogr Clin Neurophysiol Supp/ 1999; 52: 97-103.

18 Edwards DJ, Cortes M, Thickbroom GW, Rykman A, Pascual-Leone A, Volpe BT. Reply: evidence against volume conduction to explain normal MEPs in muscles with low motor power in SCl. Spinal Cord 2014; 52: 718.

19 Calancie B, Alexeeva N, Broton JG, Suys S, Hall A, Klose KJ. Distribution and latency of muscle responses to transcranial magnetic stimulation of motor cortex after spinal cord injury in humans. J Neurotrauma 1999; 16: 49-67.

20 Li K, Atkinson D, Boakye M, Tolfo CZ, Aslan S, Green M et al. Quantitative and sensitive assessment of neurophysiological status after human spinal cord injury. J Neurosurg Spine 2012; 17: 77-86.

21 Awad BI, Carmody MA, Zhang X, Lin VW, Steinmetz MP. Transcranial magnetic stimulation after spinal cord injury. World Neurosurg 2015; 83: 232-235.

22 Chye L, Nosaka K, Murray L, Edwards D, Thickbroom G. Corticomotor excitability of wrist flexor and extensor muscles during active and passive movement. Hum Mov Sci 2010; 29: 494-501.

23 Steeves JD, Lammertse D, Curt A, Fawcett JW, Tuszynski MH, Ditunno JF et al. International Campaign for Cures of Spinal Cord Injury Paralysis. Guidelines for the conduct of clinical trials for spinal cord injury (SCI) as developed by the ICCP panel: clinical trial outcome measures. Spinal Cord 2007; 45: 206-221.

24 Bunge RP, Puckett WR, Hiester ED. Observations on the pathology of several types of human spinal cord injury, with emphasis on the astrocyte response to penetrating injuries. Adv Neurol 1997; 72: 305-315.

25 Kakulas BA. Neuropathology: the foundation for new treatments in spinal cord injury. Spinal Cord 2004; 42: 549-563.

26 Tansey KE, McKay WB, Kakulas BA. Restorative neurology: consideration of the new anatomy and physiology of the injured nervous system. Clin Neurol Neurosurg 2012; 114: 436-440.

27 Hou J, Xiang Z, Yan R, Zhao M, Wu Y, Zhong J et al. Motor recovery at 6 months after admission is related to structural and functional reorganization of the spine and brain in patients with spinal cord injury. Hum Brain Mapp 2016; 37: 2195-2209.

28 Sydekum E, Ghosh A, Gullo M, Baltes C, Schwab M, Rudin M. Rapid functional reorganization of the forelimb cortical representation after thoracic spinal cord injury in adult rats. Neuroimage 2014; 87: 72-79.

29 Tandon S, Kambi N, Mohammed H, Jain N, Complete reorganization of the motor cortex of adult rats following long-term spinal cord injuries. Eur J Neurosci 2013; 38: 2271-2279. 